\title{
Common fixed point theorem for a hybrid pair of mappings in Hausdorff fuzzy metric spaces
}

\author{
M Abbas ${ }^{1}$, Basit Ali ${ }^{12^{*}}$ and A Amini-Harandi ${ }^{3,4}$
}

\section{"Correspondence:}

basit.aa@gmail.com

'Department of Mathematics, Lahore University of Management Sciences, Lahore, 54792, Pakistan ${ }^{2}$ Department of Mathematics, University of Management and Technology, C-II, Johar Town, Lahore, Pakistan Full list of author information is available at the end of the article

\begin{abstract}
In this paper, we prove a coupled fixed point theorem for a multivalued fuzzy contraction mapping in complete Hausdorff fuzzy metric spaces. As an application of the first theorem, a coupled coincidence and coupled common fixed point theorem has been proved for a hybrid pair of multivalued and single-valued mappings. It is worth mentioning that to find coupled coincidence points, we do not employ the condition of continuity of any mapping involved therein. Also, coupled coincidence points are obtained without exploiting any type of commutativity condition. Our results extend, improve, and unify some well-known results in the literature. MSC: $47 \mathrm{H} 10 ; 47 \mathrm{H0} 4$; $4 \mathrm{H} 07$
\end{abstract}

Keywords: coupled fixed point; coupled coincidence point; coupled common fixed point; $t$-norm

\section{Introduction and preliminaries}

Bhaskar and Lakshmikantham [1] introduced the concept of a coupled fixed point of a mapping $F$ from $X \times X$ to $X$ and established some coupled fixed point theorems in partially ordered sets. Later on some authors gave improved and generalized results in this context. For details, we refer to $[2,3]$.

The concept of fuzzy sets was initiated by Zadeh [4] in 1965. Fuzzy metric spaces were introduced by Kramosil and Michalek [5]. George and Veeramani [6, 7] modified the notion of fuzzy metric spaces by using continuous $t$-norm and generalized the concept of a probabilistic metric space to a fuzzy situation. Then a number of authors started the study of fixed point theory in fuzzy metric spaces; for a detailed survey, we refer to [8-18] and the references therein. Recently López and Romaguera [19] introduced a Hausdorff fuzzy metric on a set of nonempty compact subsets of a given fuzzy metric space. In 2011, Kiany et al. [20] proved fixed point and endpoint theorems for set-valued fuzzy contraction maps in fuzzy metric spaces.

Recently Abbas [21] introduced the concept of coupled fixed points of a mapping $F$ : $X \times X \rightarrow 2^{X}$ (a collection of all nonempty subsets of $X$ ) and coupled coincidence points of a hybrid pair $F$ and $g: X \rightarrow X$. The aim of this paper is to obtain a coupled fixed point theorem for $F$ and a coupled coincidence and coupled common fixed point theorem for a hybrid pair $\{F, g\}$ which satisfies a contractive condition in complete Hausdorff fuzzy metric spaces. It is to be noted that to find coupled coincidence points, we do not employ the

(c) 2012 Abbas et al.: licensee Springer. This is an Open Access article distributed under the terms of the Creative Commons Attribution License (http://creativecommons.org/licenses/by/2.0), which permits unrestricted use, distribution, and reproduction in any medium, provided the original work is properly cited. 
condition of commutativity and continuity of any mapping involved therein. Our results unify, extend, and generalize various known comparable results given in existing literature (see, for example, [20] and some references therein).

Definition $1[22]$ A binary operation $*:[0,1]^{2} \rightarrow[0,1]$ is called a continuous $t$-norm if

(1) $*$ is associative and commutative;

(2) $*$ is continuous;

(3) $a * 1=a$ for all $a \in[0,1]$;

(4) $a * b \leq c * d$ whenever $a \leq c$ and $b \leq d$.

Definition 2 [6] Let $X$ be a nonempty set and $*$ be a continuous $t$-norm. If a mapping $M: X^{2} \times[0, \infty) \rightarrow[0,1]$ satisfies the following conditions:

(F1) $M(x, y, t)>0$;

(F2) $M(x, y, t)=1$ if and only if $x=y$;

(F3) $M(x, y, t)=M(y, x, t)$;

(F4) $M(x, y, t) * M(y, z, s) \leq M(x, z, t+s)$;

(F5) $M(x, y, t):(0, \infty) \rightarrow[0,1]$ is continuous;

for each $x, y, z \in X$ and $s, t>0$, then 3 -tuple $(X, M, *)$ is called a fuzzy metric space.

Example $3[6]$ Let $(X, d)$ be a metric space. Define $a * b=\min \{a, b\}$ and

$$
M(x, y, t)=\frac{t}{t+d(x, y)}
$$

for all $x, y \in X$ and $t>0$. Then $(X, M, *)$ is a fuzzy metric space. We call this a fuzzy metric $M$, the standard fuzzy metric induced by $d$.

Definition 4 [6] Let $(X, M, *)$ be a fuzzy metric space.

(i) A sequence $\left\{x_{n}\right\}$ is said to be convergent to a point $x \in X$ if $\lim _{n \rightarrow \infty} M\left(x_{n}, x, t\right)=1$ for all $t>0$.

(ii) A sequence $\left\{x_{n}\right\}$ is said to be a Cauchy sequence if $\lim _{n \rightarrow \infty} M\left(x_{m}, x_{n}, t\right)=1$ for all $t>0$.

(iii) A fuzzy metric space in which every Cauchy sequence is convergent is said to be complete.

(iv) A subset $A \subseteq X$ is said to be closed if for each convergent sequence $\left\{x_{n}\right\}$ with $x_{n} \in A$ and $x_{n} \rightarrow x$, we have $x \in A$.

(v) A subset $A \subseteq X$ is said to be compact if each sequence in $A$ has a convergent subsequence. The set of all compact subsets of $X$ will be denoted by $K(X)$.

Lemma 5 [10] For all $x, y \in X, M(x, y, \cdot)$ is nondecreasing.

Definition 6 Let $(M, X, *)$ be a fuzzy metric space, $M$ is said to be continuous on $X^{2} \times$ $(0, \infty)$ if

$$
\lim _{n \rightarrow \infty} M\left(x_{n}, y_{n}, t_{n}\right)=M(x, y, t)
$$


whenever $\left\{\left(x_{n}, y_{n}, t_{n}\right)\right\}$ is a sequence in $X^{2} \times(0, \infty)$ which converges to a point $(x, y, t) \in$ $X^{2} \times(0, \infty)$; that is,

$$
\lim _{n \rightarrow \infty} M\left(x_{n}, x, t\right)=\lim _{n \rightarrow \infty} M\left(y_{n}, y, t\right)=1 \quad \text { and } \quad \lim _{n \rightarrow \infty} M\left(x, y, t_{n}\right)=M(x, y, t) .
$$

Lemma 7 [10] $M$ is a continuous function on $X^{2} \times(0, \infty)$.

Kiany et al. [20] introduced the following lemma in fuzzy metric spaces.

Lemma 8 [20] Let $(X, M, *)$ be a fuzzy metric space satisfying

$$
\lim _{n \rightarrow \infty} *_{i=n}^{\infty} M\left(x, y, t h^{i}\right)=1
$$

for every $x, y \in X, t>0$, and $h>1$. Suppose $\left\{x_{n}\right\}$ is a sequence in $X$ satisfying

$$
M\left(x_{n}, x_{n+1}, \alpha t\right) \geq M\left(x_{n-1}, x_{n}, t\right)
$$

for all $n \in \mathbb{N}$ and $0<\alpha<1$. Then $\left\{x_{n}\right\}$ is a Cauchy sequence.

Lemma 9 [19] Let $(X, M, *)$ be a fuzzy metric space. Then, for each $a \in X, B \in K(X)$, and $t>0$, there is a $b_{0} \in B$ such that $M(a, B, t)=M\left(a, b_{0}, t\right)$, where

$$
M(a, B, t)=\sup _{b \in B} M(a, b, t) .
$$

Definition 10 [19] Let $(X, M, *)$ be a fuzzy metric space. For each $A, B \in K(X)$ and $t>0$, set

$$
H_{M}(A, B, t)=\min \left\{\inf _{x \in A} M(x, B, t), \inf _{y \in B} M(A, y, t)\right\} .
$$

The 3-tuple $\left(K(X), H_{M}, *\right)$ is called a Hausdorff fuzzy metric space.

Lemma 11 [23] Let $X$ be a nonempty set and $g: X \rightarrow X$ be a mapping. Then there exists a subset $E \subseteq X$ such that $g(E)=g(X)$ and $g: E \rightarrow X$ is one-to-one.

Theorem 12 [20] Let $(X, M, *)$ be a complete fuzzy metric. Suppose $F: X \times X \rightarrow K(X)$ is a multivalued mapping such that

$$
H_{M}(F x, F y, \phi(d(x, y, t)) t) \geq M(x, y, t)
$$

for each $x, y \in X$ and $t>0$, where $\phi:[0, \infty) \rightarrow[0,1]$ satisfying $\lim \sup _{r \rightarrow t^{+}} \phi(r)<1$, for all $t \in[0, \infty)$, and $d(x, y, t)=\frac{t}{M(x, y, t)}-t$. Furthermore, assume that $(X, M, *)$ satisfies (1) for some $x_{0}$ and $x_{1} \in F\left(x_{0}\right)$. Then $F$ has a fixed point.

We also need the following definitions given in [21].

Definition 13 [21] Let $X$ be a nonempty set, $F: X \times X \rightarrow 2^{X}$ (a collection of all nonempty subsets of $X)$ and $g: X \rightarrow X$. An element $(x, y) \in X \times X$ is called

(C1) a coupled fixed point of $F$ if $x \in F(x, y)$ and $y \in F(y, x)$; 
(C2) a coupled coincidence point of a hybrid pair $\{F, g\}$ if $g(x) \in F(x, y)$ and $g(y) \in F(y, x)$

(C3) a coupled common fixed point of a hybrid pair $\{F, g\}$ if $x=g(x) \in F(x, y)$ and $y=g(y) \in F(y, x)$.

We denote the set of coupled coincidence points of mappings $F$ and $g$ by $C(F, g)$. Note that if $(x, y) \in C(F, g)$, then $(y, x)$ is also in $C(F, g)$.

Definition 14 [21] Let $F: X \times X \rightarrow 2^{X}$ be a multivalued mapping and $g$ be a self-map on $X$. The hybrid pair $\{F, g\}$ is called $w$-compatible if $g(F(x, y)) \subseteq F(g x, g y)$ whenever $(x, y) \in$ $C(F, g)$.

Definition 15 [21] Let $F: X \times X \rightarrow 2^{X}$ be a multivalued mapping and $g$ be a self-mapping on $X$. The mapping $g$ is called $F$-weakly commuting at some point $(x, y) \in X \times X$ if $g^{2}(x) \in$ $F(g x, g y)$ and $g^{2}(y) \in F(g y, g x)$.

\section{Coupled fixed and coincidence point theorems}

In the following theorem, we obtain a coupled fixed point for a multivalued mapping satisfying a contractive condition.

Theorem 16 Let $(X, M, *)$ be a complete fuzzy metric space and let $F: X \times X \rightarrow K(X)$ be a set-valued mapping satisfying

$$
H_{M}(F(x, y), F(u, v), \phi(d(x, u, t)) t) \geq \min \{M(x, u, t), M(x, F(x, y), t)\}
$$

for each $x, y, u, v \in X, t>0$. Suppose that $d(x, u, t)=\frac{t}{M(x, u, t)}-t$ and $\phi:[0, \infty) \rightarrow[0,1)$ is a mapping satisfying

$$
\limsup \phi(r)<1
$$

for all $t \in[0, \infty)$. Furthermore, assume that $(X, M, *)$ satisfies (1) for some $x_{0}, x_{1} \in F\left(x_{0}, y_{0}\right)$ and $y_{0}, y_{1} \in F\left(y_{0}, x_{0}\right)$. Then $F$ has a coupled fixed point.

Proof Let $x_{0}, y_{0} \in X$ be arbitrary. Choose $x_{1} \in F\left(x_{0}, y_{0}\right)$ and $y_{1} \in F\left(y_{0}, x_{0}\right)$. Since $F$ is compact valued, then by Lemma 9 there exists $x_{2} \in F\left(x_{1}, y_{1}\right)$ such that

$$
\begin{aligned}
M\left(x_{1}, x_{2}, t\right) & \geq M\left(x_{1}, x_{2}, \phi\left(d\left(x_{0}, x_{1}, t\right)\right) t\right) \\
& =\sup _{y \in F\left(x_{1}, y_{1}\right)} M\left(x_{1}, y, \phi\left(d\left(x_{0}, x_{1}, t\right)\right) t\right) \\
& \geq H_{M}\left(F\left(x_{0}, y_{0}\right), F\left(x_{1}, y_{1}\right), \phi\left(d\left(x_{0}, x_{1}, t\right)\right) t\right) \\
& \geq \min \left\{M\left(x_{0}, x_{1}, t\right), M\left(x_{0}, F\left(x_{0}, y_{0}\right), t\right)\right\} \geq M\left(x_{0}, x_{1}, t\right) .
\end{aligned}
$$

Since $F$ is compact valued, there exists $y_{2} \in F\left(y_{1}, x_{1}\right)$ such that

$$
\begin{aligned}
M\left(y_{1}, y_{2}, t\right) & \geq M\left(y_{1}, y_{2}, \phi\left(d\left(y_{0}, y_{1}, t\right)\right) t\right) \\
& =\sup _{y \in F\left(y_{1}, x_{1}\right)} M\left(y_{1}, y, \phi\left(d\left(y_{0}, y_{1}, t\right)\right) t\right)
\end{aligned}
$$




$$
\begin{aligned}
& \geq H_{M}\left(F\left(y_{0}, x_{0}\right), F\left(y_{1}, x_{1}\right), \phi\left(d\left(y_{0}, y_{1}, t\right)\right) t\right) \\
& \geq \min \left\{M\left(y_{0}, y_{1}, t\right), M\left(y_{0}, F\left(y_{0}, x_{0}\right), t\right)\right\} \geq M\left(y_{0}, y_{1}, t\right) .
\end{aligned}
$$

Continuing this process, we obtain a sequence $\left\{x_{n}\right\}_{n \geq 0}$ and $\left\{y_{n}\right\}_{n \geq 0}$ in $X$ such that $x_{n+1} \in$ $F\left(x_{n}, y_{n}\right)$ and $y_{n+1} \in F\left(y_{n}, x_{n}\right)$ satisfying

$$
\begin{aligned}
M\left(x_{n}, x_{n+1}, t\right) & \geq M\left(x_{n}, x_{n+1}, \phi\left(d\left(x_{n-1}, x_{n}, t\right)\right) t\right) \\
& =\sup _{y \in F\left(x_{n}, y_{n}\right)} M\left(x_{n}, y, \phi\left(d\left(x_{n-1}, x_{n}, t\right)\right) t\right) \\
& \geq H_{M}\left(F\left(x_{n-1}, y_{n-1}\right), F\left(x_{n}, y_{n}\right), \phi\left(d\left(x_{n-1}, x_{n}, t\right)\right) t\right) \\
& \geq \min \left\{M\left(x_{n-1}, x_{n}, t\right), M\left(x_{n-1}, F\left(x_{n-1}, y_{n-1}\right), t\right)\right\} \geq M\left(x_{n-1}, x_{n}, t\right) .
\end{aligned}
$$

That is, we obtain

$$
M\left(x_{n}, x_{n+1}, t\right) \geq M\left(x_{n-1}, x_{n}, t\right) .
$$

Similarly,

$$
M\left(y_{n}, y_{n+1}, t\right) \geq M\left(y_{n-1}, y_{n}, t\right) .
$$

Inequalities (3) and (4) show that the sequences $\left\{M\left(x_{n}, x_{n+1}, t\right)\right\}_{n}$ and $\left\{M\left(y_{n}, y_{n+1}, t\right)\right\}_{n}$ are nondecreasing. Thus, $d\left(x_{n}, x_{n+1}, t\right)$ and $d\left(y_{n}, y_{n+1}, t\right)$ are nonnegative nonincreasing and so they are convergent, say, to $l_{1} \geq 0$ and $l_{2} \geq 0$. Since, by the given assumption,

$$
\begin{aligned}
& \limsup _{n \rightarrow \infty} \phi\left(d\left(x_{n}, x_{n+1}, t\right)\right) \leq \underset{r \rightarrow l_{1}^{+}}{\limsup \phi(r)<1 \quad \text { and }} \\
& \limsup _{n \rightarrow \infty} \phi\left(d\left(y_{n}, y_{n+1}, t\right)\right) \leq \underset{r \rightarrow l_{2}^{+}}{\limsup \phi(r)<1,}
\end{aligned}
$$

then there exist $k_{1}<1, k_{2}<1$, and $N_{1}, N_{2} \in \mathbb{N}$ such that

$$
\begin{array}{ll}
\phi\left(d\left(x_{n}, x_{n+1}, t\right)\right)<k_{1} & \text { for all } n>N_{1} \quad \text { and } \\
\phi\left(d\left(y_{n}, y_{n+1}, t\right)\right)<k_{2} & \text { for all } n>N_{2} .
\end{array}
$$

Since $M(x, y, \cdot)$ is nondecreasing, then (3), (4), and (6) yield

$$
\begin{aligned}
& M\left(x_{n}, x_{n+1}, k_{1} t\right) \geq M\left(x_{n}, x_{n+1}, \phi\left(d\left(x_{n-1}, x_{n}, t\right)\right) t\right) \geq M\left(x_{n-1}, x_{n}, t\right) \quad \text { and } \\
& M\left(y_{n}, y_{n+1}, k_{2} t\right) \geq M\left(y_{n}, y_{n+1}, \phi\left(d\left(y_{n-1}, y_{n}, t\right)\right) t\right) \geq M\left(y_{n-1}, y_{n}, t\right) .
\end{aligned}
$$

Then we obtain

$$
\begin{aligned}
& M\left(x_{n}, x_{n+1}, k_{1} t\right) \geq M\left(x_{n-1}, x_{n}, t\right) \quad \text { and } \\
& M\left(y_{n}, y_{n+1}, k_{2} t\right) \geq M\left(y_{n-1}, y_{n}, t\right) .
\end{aligned}
$$


Hence, by Lemma $8,\left\{x_{n}\right\}$ and $\left\{y_{n}\right\}$ are Cauchy sequences. Since $(X, M, *)$ is a complete fuzzy metric space, then there exist $x$ and $y$ in $X$ such that $\lim _{n \rightarrow \infty} x_{n}=x$ and $\lim _{n \rightarrow \infty} y_{n}=$ $y$, we have

$$
\begin{array}{lll}
\lim _{n \rightarrow \infty} M\left(x_{n}, x, t\right)=1 & \text { for each } t>0 \quad \text { and } \\
\lim _{n \rightarrow \infty} M\left(y_{n}, y, t\right)=1 & \text { for each } t>0 .
\end{array}
$$

Thus

$$
\begin{array}{lll}
\lim _{n \rightarrow \infty} d\left(x_{n}, x, t\right)=0 & \text { for each } t>0 \quad \text { and } \\
\lim _{n \rightarrow \infty} d\left(y_{n}, y, t\right)=0 & \text { for each } t>0 .
\end{array}
$$

Since

$$
\begin{aligned}
& \limsup _{n \rightarrow \infty} \phi\left(d\left(x_{n}, x, t\right)\right) \leq \underset{r \rightarrow 0^{+}}{\limsup \phi(r)<1 \quad \text { and }} \\
& \limsup _{n \rightarrow \infty} \phi\left(d\left(y_{n}, y, t\right)\right) \leq \underset{r \rightarrow 0^{+}}{\limsup \phi(r)<1,}
\end{aligned}
$$

then there exist $\lambda_{1}, \lambda_{2}$ such that $\lambda_{1}<1, \lambda_{2}<1$, and $n_{1}, n_{2} \in \mathbb{N}$ such that

$$
\begin{array}{ll}
\phi\left(d\left(x_{n}, x, t\right)\right)<\lambda_{1} & \text { for all } n>n_{1} \quad \text { and } \\
\phi\left(d\left(y_{n}, y, t\right)\right)<\lambda_{2} & \text { for all } n>n_{2} .
\end{array}
$$

Now, we show that $x \in F(x, y)$ and $y \in F(y, x)$. Consider

$$
\begin{aligned}
H_{M}\left(F\left(x_{n}, y_{n}\right), F(x, y), \lambda_{1} t\right) & \geq H_{M}\left(F\left(x_{n}, y_{n}\right), F(x, y), \phi\left(d\left(x_{n}, x, t\right)\right) t\right) \\
& \geq \min \left\{M\left(x_{n}, x, t\right), M\left(x_{n}, F\left(x_{n}, y_{n}\right), t\right)\right\} \\
& \geq \min \left\{M\left(x_{n}, x, t\right), M\left(x_{n}, x_{n+1}, t\right)\right\} \\
& \geq \min \left\{M\left(x_{n}, x, t\right), M\left(x_{n}, x, \frac{t}{2}\right) * M\left(x, x_{n+1}, \frac{t}{2}\right)\right\} .
\end{aligned}
$$

On taking limit as $n \rightarrow \infty$, we obtain

$$
\lim _{n \rightarrow \infty} H_{M}\left(F\left(x_{n}, y_{n}\right), F(x, y), \lambda_{1} t\right)=1 \text {. }
$$

So that

$$
\lim _{n \rightarrow \infty} H_{M}\left(F\left(x_{n}, y_{n}\right), F(x, y), t\right)=1 \text {. }
$$

Similarly, we can obtain

$$
\lim _{n \rightarrow \infty} H_{M}\left(F\left(y_{n}, x_{n}\right), F(y, x), t\right)=1
$$


Since $x_{n+1} \in F\left(x_{n}, y_{n}\right)$ and $y_{n+1} \in F\left(y_{n}, x_{n}\right)$, from (7) and (8) we obtain

$$
\lim _{n \rightarrow \infty} \sup _{a \in F(x, y)} M\left(x_{n+1}, a, t\right)=1 \quad \text { and } \quad \lim _{n \rightarrow \infty} \sup _{b \in F(y, x)} M\left(y_{n+1}, b, t\right)=1 .
$$

There exist sequences $w_{n} \in F(x, y)$ and $z_{n} \in F(y, x)$ such that

$$
\lim _{n \rightarrow \infty} M\left(x_{n+1}, w_{n+1}, t\right)=1 \quad \text { and } \quad \lim _{n \rightarrow \infty} M\left(y_{n+1}, z_{n+1}, t\right)=1
$$

for each $t>0$. Now, for each $n \in \mathbb{N}$, we have

$$
M\left(w_{n+1}, x, t\right) \geq M\left(w_{n+1}, x_{n+1}, \frac{t}{2}\right) * M\left(x_{n+1}, x, \frac{t}{2}\right) .
$$

On taking limit as $n \rightarrow \infty$, we get

$$
\lim _{n \rightarrow \infty} M\left(w_{n+1}, x, t\right)=1 .
$$

Similarly, for each $n \in \mathbb{N}$, we have

$$
M\left(z_{n+1}, y, t\right) \geq M\left(z_{n+1}, y_{n+1}, \frac{t}{2}\right) * M\left(y_{n+1}, y, \frac{t}{2}\right) .
$$

On taking limit as $n \rightarrow \infty$, we get

$$
\lim _{n \rightarrow \infty} M\left(z_{n+1}, y, t\right)=1
$$

(9) and (10) imply

$$
\lim _{n \rightarrow \infty} w_{n}=x \text { and } \quad \lim _{n \rightarrow \infty} z_{n}=y .
$$

Since $F(x, y)$ and $F(y, x)$ are compact, we get $x \in F(x, y)$ and $y \in F(y, x)$.

Corollary 17 Let $(X, M, *)$ be a complete fuzzy metric space and let $F: X \times X \rightarrow K(X)$ be a mapping satisfying

$$
H_{M}(F(x, y), F(u, v), k t) \geq \min \{M(x, u, t), M(x, F(x, y), t)\}
$$

for each $x, y, u, v \in X, t>0$, and $0<k<1$. Suppose that $(X, M, *)$ satisfies $(1)$ for some $x_{0}, x_{1} \in$ $F\left(x_{0}, y_{0}\right)$ and $y_{0}, y_{1} \in F\left(y_{0}, x_{0}\right)$. Then $F$ has a coupled fixed point.

Corollary 18 Let $(X, M, *)$ be a complete fuzzy metric space and let $F: X \times X \rightarrow K(X)$ be a set-valued mapping satisfying

$$
H_{M}(F(x, y), F(u, v), \phi(d(x, u, t)) t) \geq M(x, u, t)
$$

for each $x, y, u, v \in X, t>0$. Suppose that $d(x, u, t)=\frac{t}{M(x, u, t)}-t$ and $\phi:[0, \infty) \rightarrow[0,1)$ is a mapping satisfying

$$
\limsup _{r \rightarrow t^{+}} \phi(r)<1
$$


for all $t \in[0, \infty)$. Furthermore, assume that $(X, M, *)$ satisfies (1) for some $x_{0}, x_{1} \in F\left(x_{0}, y_{0}\right)$ and $y_{0}, y_{1} \in F\left(y_{0}, x_{0}\right)$. Then $F$ has a coupled fixed point.

Corollary 19 Let $(X, M, *)$ be a complete fuzzy metric space and let $F: X \times X \rightarrow K(X)$ be a mapping satisfying

$$
H_{M}(F(x, y), F(u, v), k t) \geq M(x, u, t)
$$

for each $x, y, u, v \in X, t>0$, and $0<k<1$. Suppose that $(X, M, *)$ satisfies (1) for some $x_{0}, x_{1} \in$ $F\left(x_{0}, y_{0}\right)$ and $y_{0}, y_{1} \in F\left(y_{0}, x_{0}\right)$. Then $F$ has a coupled fixed point.

Example 20 Let $X=\{0,1,2\}$ and $d: X \times X \rightarrow R$ be defined as

$$
\begin{aligned}
& d(0,1)=2, \quad d(1,2)=1.5, \quad d(0,2)=1, \\
& d(0,0)=d(1,1)=d(2,2)=0, \\
& d(a, b)=d(b, a) \quad \text { for all } a, b \in X .
\end{aligned}
$$

Hence, $(X, d)$ is a metric space. Consider

$$
M(x, y, t)=\frac{t}{t+d(x, y)}
$$

for $t>0$ and $(X, M, *)$ satisfies (1). Define $F: X \times X \rightarrow C B(X)$ as follows:

$$
F(x, y)= \begin{cases}\{0,2\} & \text { when } x \neq 1 \text { and } y \in X, \\ \{0\} & \text { when } x=1 \text { and } y \in X .\end{cases}
$$

For $x \in\{0,2\}$ and $u \in\{0,2\}$ and for $x=1$ and $u=1$, we have

$$
H_{M}(F(x, y), F(u, v), k t)=1
$$

for any $k \in(0,1)$. Hence, (11) is satisfied. For the rest of cases,

$$
H_{M}(F(x, y), F(u, v), k t)=\frac{k t}{k t+1}
$$

and

$$
\min \{M(x, u, t), M(x, F(x, y), t)\} \in\left\{0, \frac{t}{t+2}, \frac{t}{t+1.5}\right\} .
$$

Hence, for all $x, y, u, v \in X, t>0$, and $k=\frac{2}{3}$, (11) holds. All the conditions of Corollary 17 and Theorem 16 with $\phi(t)=k$ are satisfied. Moreover, $(0,0)$ and $(2,2)$ are coupled fixed points of $F$.

Example 21 Let $X=[0,1]$ be endowed with the usual metric $d(x, y)=|x-y|$ and $F(x, y)=$ $[\ln (1+x), 1]$ for each $x, y \in X$. Let $M(x, y, t)=\frac{t}{t+d(x, y)}$ and let $\phi(t)=\frac{\ln (1+t)}{t}$ for each $t>0$. Then 
we have

$$
\begin{aligned}
H_{M}(F(x, y), F(u, v), \phi(t) t) & =H_{M}(F(x, y), F(u, v), t) \\
& \geq \frac{t}{t+|\ln (1+u)-\ln (1+x)|} \\
& \geq \frac{t}{t+|u-x|} \\
& =M(x, u, t)
\end{aligned}
$$

for each $x, y \in X$ and $t>0$. Then by Corollary $18, F$ has a coupled fixed point $((0,0)$ is a coupled fixed point of $F$ ).

Example 22 Let $X=R$ be endowed with the usual metric $d(x, y)=|x-y|$ and let $f(x, y)=$ $\frac{1}{2} x$ for each $x, y \in X$. Let $M(x, y, t)=\frac{t}{t+d(x, y)}$ and let $\phi(t)=\frac{1}{2}$ for each $t>0$. Then we have

$$
\begin{aligned}
H_{M}\left(f(x, y), f(u, v), \frac{1}{2} t\right) & =M\left(f(x, y), f(u, v), \frac{1}{2} t\right) \\
& =M\left(\frac{1}{2} x, \frac{1}{2} u, \frac{1}{2} t\right)=M(x, u, t)
\end{aligned}
$$

for each $x, y \in X$ and $t>0$. Then by Corollary $19, f$ has a coupled fixed point $((0,0)$ is a coupled fixed point of $f$ ).

Now, as an application of the above theorem, we obtain a coupled coincidence and common fixed point theorem for a hybrid pair of multivalued and single-valued mappings.

Theorem 23 Let $(X, M, *)$ be a complete fuzzy metric space and let $F: X \times X \rightarrow K(X)$ and $g: X \rightarrow X$ be mappings satisfying

$$
H_{M}(F(x, y), F(u, v), \phi(d(x, u, t)) t) \geq \min \{M(g x, g u, t), M(g x, F(g x, g y), t)\}
$$

for each $x, y, u, v \in X, t>0$. Suppose that $d(x, u, t)=\frac{t}{M(x, u, t)}-t$ and $\phi:[0, \infty) \rightarrow[0,1)$ is a mapping satisfying

$$
\limsup _{r \rightarrow t^{+}} \phi(r)<1
$$

for all $t \in[0, \infty)$. Furthermore, assume that $F(X \times X) \subseteq g(X)$ and $(X, M, *)$ satisfies (1) for some $g x_{0}, g x_{1} \in F\left(x_{0}, y_{0}\right)$ and $g y_{0}, g y_{1} \in F\left(y_{0}, x_{0}\right)$. Then $F$ and $g$ have a coupled coincidence point. Moreover, $F$ and $g$ have a coupled common fixed point if one of the following conditions holds:

(a) $F$ and $g$ are $w$-compatible, $\lim _{n \rightarrow \infty} g^{n} x=u$ and $\lim _{n \rightarrow \infty} g^{n} y=v$ for some $(x, y) \in C(F, g), u, v \in X$, and $g$ is continuous at $u$ and $v$.

(b) $g$ is $F$-weakly commuting for some $(x, y) \in C(g, F)$, and $g x$ and gy are fixed points of $g$, that is, $g^{2} x=g x$ and $g^{2} y=g y$.

(c) $g$ is continuous at $x, y$ for some $(x, y) \in C(g, F)$ and for some $u, v \in X, \lim _{n \rightarrow \infty} g^{n} u=x$ and $\lim _{n \rightarrow \infty} g^{n} v=y$. 
Proof By Lemma 11 there exists $E \subseteq X$ such that $g: E \rightarrow X$ is one-to-one and $g(E)=g(X)$. Now, define a mapping $\mathcal{A}: g(E) \times g(E) \rightarrow K(X)$ by

$$
\mathcal{A}(g x, g y)=F(x, y) \quad \text { for all } g x, g y \in g(E)
$$

and since $g$ is one-one on $E$, so $\mathcal{A}$ is well defined. Further,

$$
\begin{aligned}
H_{M}(\mathcal{A}(g x, g y), \mathcal{A}(g u, g v), \phi(d(x, u, t)) t) & =H_{M}(F(x, y), F(u, v), \phi(d(x, u, t)) t) \\
& \geq \min \{M(g x, g u, t), M(g x, F(g x, g y), t)\} .
\end{aligned}
$$

Hence, $\mathcal{A}$ satisfies (2) and all the conditions of Theorem 16. By using Theorem 16 with a mapping $\mathcal{A}$, it follows that $\mathcal{A}$ has a coupled fixed point $(u, v) \in g(E) \times g(E)$. Finally, it is left to prove that $F$ and $g$ have a coupled coincidence point. Since $\mathcal{A}$ has a coupled fixed point $(u, v) \in g(E) \times g(E)$, we get

$$
u \in \mathcal{A}(u, v), \quad \text { and } \quad v \in \mathcal{A}(v, u) \text {. }
$$

Since $F(X \times X) \subseteq g(X)$, so there exist $u_{1}, v_{1} \in X \times X$ such that $g u_{1}=u$ and $g v_{1}=v$. Thus, it follows from (15),

$$
\begin{aligned}
& g u_{1} \in \mathcal{A}\left(g u_{1}, g v_{1}\right)=F\left(u_{1}, v_{1}\right), \\
& g v_{1} \in \mathcal{A}\left(g v_{1}, g u_{1}\right)=F\left(v_{1}, u_{1}\right) .
\end{aligned}
$$

This implies that $\left(u_{1}, v_{1}\right) \in X \times X$ is a coupled coincidence point of $F$ and $g$. Hence, $C(F, g)$ is nonempty. Suppose now that (a) holds. Then, for some $(x, y) \in C(F, g)$,

$$
\lim _{n \rightarrow \infty} g^{n} x=u \text { and } \quad \lim _{n \rightarrow \infty} g^{n} y=v
$$

where $u, v \in X$. Since $g$ is continuous at $u$ and $v$, we have that $u$ and $v$ are fixed points of $g$. As $F$ and $g$ are $w$-compatible, so

$$
\left(g^{n} x, g^{n} y\right) \in C(F, g) \quad \text { for all } n \geq 1 \text {. }
$$

That is, for all $n \geq 1$,

$$
\begin{aligned}
& g^{n} x \in F\left(g^{n-1} x, g^{n-1} y\right) \quad \text { and } \\
& g^{n} y \in F\left(g^{n-1} y, g^{n-1} x\right) .
\end{aligned}
$$

Using (12), we obtain

$$
\begin{aligned}
& M(g u, F(u, v), t) \\
& \quad \geq M\left(g u, g^{n} x, \frac{t}{2}\right) * M\left(g^{n} x, F(u, v), \frac{t}{2}\right) \\
& \quad \geq M\left(g u, g^{n} x, \frac{t}{2}\right) * H_{M}\left(F\left(g^{n-1} x, g^{n-1} y\right), F(u, v), \frac{t}{2}\right)
\end{aligned}
$$




$$
\begin{aligned}
& \geq M\left(g u, g^{n} x, \frac{t}{2}\right) * \min \left\{\left(M\left(g^{n} x, g u, \frac{t}{2}\right)\right), M\left(g^{n} x, F\left(g^{n-1} x, g^{n-1} y\right), \frac{t}{2}\right)\right\} \\
& \leq M\left(g u, g^{n} x, \frac{t}{2}\right) * \min \left\{\left(M\left(g^{n} x, g u, \frac{t}{2}\right)\right), M\left(g^{n} x, g^{n} x, \frac{t}{2}\right)\right\} \\
& \geq M\left(g u, g^{n} x, \frac{t}{2}\right) * M\left(g^{n} x, g u, \frac{t}{2}\right) .
\end{aligned}
$$

On taking limit as $n \rightarrow \infty$, we get

$$
\begin{aligned}
M(g u, F(u, v), t) & \geq M\left(u, u, \frac{t}{2}\right) * M\left(u, u, \frac{t}{2}\right) \\
& \geq 1 * 1=1 .
\end{aligned}
$$

This implies $g u \in F(u, v)$. Similarly, $g v \in F(v, u)$. Consequently, $u=g u \in F(u, v)$ and $v=$ $g v \in F(v, u)$. Hence, $(u, v)$ is a coupled common fixed point of $F$ and $g$. Suppose now that (b) holds. If for some $(x, y) \in C(F, g), g$ is $F$-commuting and $g^{2} x=g x$ and $g^{2} y=g y$, then

$$
\begin{aligned}
& g x=g^{2} x \in F(g x, g y) \quad \text { and } \\
& g y=g^{2} y \in F(g y, g x) .
\end{aligned}
$$

Hence, $(g x, g y)$ is a coupled common fixed point of $F$ and $g$. Suppose now that (c) holds and assume that for some $(x, y) \in C(g, F)$ and for some $u, v \in X, \lim _{n \rightarrow \infty} g^{n} u=x$ and $\lim _{n \rightarrow \infty} g^{n} v=y$. By the continuity of $g$ at $x$ and $y$, we get

$$
\begin{aligned}
& x=g x \in F(x, y) \quad \text { and } \\
& y=g y \in F(y, x) .
\end{aligned}
$$

Hence, $(x, y)$ is a coupled common fixed point of $F$ and $g$.

\section{Competing interests}

The authors declare that they have no competing interests.

\section{Authors' contributions}

All authors read and approved the final manuscript.

\section{Author details}

'Department of Mathematics, Lahore University of Management Sciences, Lahore, 54792, Pakistan. ${ }^{2}$ Department of Mathematics, University of Management and Technology, C-II, Johar Town, Lahore, Pakistan. ${ }^{3}$ Department of Mathematics, University of Shahrekord, Shahrekord, 88186-34141, Iran. ${ }^{4}$ School of Mathematics, Institute for Research in Fundamental Sciences (IPM), P.O. Box 19395-5746, Tehran, Iran.

\section{Acknowledgements}

The authors would like to thank the editor and anonymous reviewers for their helpful comments that helped to improve the presentation of this paper. The third author was partially supported by the Center of Excellence for Mathematics, University of Shahrekord, Iran and by a grant from IPM (No. 91470412).

Received: 24 April 2012 Accepted: 25 November 2012 Published: 13 December 2012

\section{References}

1. Bhashkar, TG, Lakshmikantham, V: Fixed point theorems in partially ordered metric spaces and applications. Nonlinear Anal. TMA 65, 1379-1393 (2006)

2. Lakshmikantham, V, Ćirić, L: Coupled fixed point theorems for nonlinear contractions in partially ordered metric space. Nonlinear Anal. TMA 70, 4341-4349 (2009) 
3. Sabetghadam, F, Masiha, HP, Sanatpour, AH: Some coupled fixed point theorems in cone metric space. Fixed Point Theory Appl. 2009, Article ID 125426 (2009)

4. Zadeh, LA: Fuzzy sets. Inf. Control 8, 103-112 (1965)

5. Kramosil, I, Michalek, J: Fuzzy metric and statistical metric spaces. Kybernetika 11, 326-334 (1975)

6. George, A, Veeramani, P: On some results in fuzzy metric spaces. Fuzzy Sets Syst. 64, 395-399 (1994)

7. George, A, Veeramani, P: On some results of analysis for fuzzy metric spaces. Fuzzy Sets Syst. 90, 365-368 (1997)

8. Ray, AD, Saha, PK: Fixed point theorems on generalized fuzzy metric spaces. Hacet. J. Math. Stat. 39, 1-9 (2010)

9. Fang, JX: On fixed point theorems in fuzzy metric spaces. Fuzzy Sets Syst. 46, 107-113 (1992)

10. Grabiec, M: Fixed points in fuzzy metric spaces. Fuzzy Sets Syst. 27, 385-389 (1983)

11. Gregori, V, Sapena, A: On fixed-point theorems in fuzzy metric spaces. Fuzzy Sets Syst. 125, 245-252 (2002)

12. Liu, Y, Li, Z: Coincidence point theorems in probabilistic and fuzzy metric spaces. Fuzzy Sets Syst. 158, 58-70 (2007)

13. Miheț, D: On the existence and the uniqueness of fixed points of Sehgal contractions. Fuzzy Sets Syst. 156, 135-141 (2005)

14. Miheţ, D: On fuzzy contractive mappings in fuzzy metric spaces. Fuzzy Sets Syst. 158, 915-921 (2007)

15. Razani, A: A contraction theorem in fuzzy metric space. Fixed Point Theory Appl. 3, 257-265 (2005)

16. Sedghi, S, Altun, I, Shobe, N: Coupled fixed point theorems for contractions in fuzzy metric spaces. Nonlinear Anal. 72, 1298-1304 (2010)

17. Som, T, Mukherjee, RN: Some fixed point theorems for fuzzy mappings. Fuzzy Sets Syst. 33, 213-219 (1989)

18. Žikić, T: On fixed point theorems of Gregori and Sapena. Fuzzy Sets Syst. 144, 421-429 (2004)

19. Rodríguez-López, J, Romaguera, S: The Hausdorff fuzzy metric on compact sets. Fuzzy Sets Syst. 147, 273-283 (2004)

20. Kiany, F, Amini-Harandi, A: Fixed point and endpoint theorems for set-valued fuzzy contraction maps in fuzzy metric spaces. Fixed Point Theory Appl. 2011, 94 (2011). doi:10.1186/1687-1812-2011-94

21. Abbas, M, Ćirić, L, Damjanović, B, Khan, MA: Coupled coincidence and common fixed point theorems for hybrid pair of mappings. Fixed Point Theory Appl. (2012). doi:10.1186/1687-1812-2012-4

22. Schweizer, B, Sklar, A: Statistical metric spaces. Pac. J. Math. 10, 314-334 (1960)

23. Haghi, RH, Rezapour, SH, Shahzad, N: Some fixed point generalizations are not real generalizations. Nonlinear Anal. 74, 1799-1803 (2011)

doi:10.1186/1687-1812-2012-225

Cite this article as: Abbas et al.: Common fixed point theorem for a hybrid pair of mappings in Hausdorff fuzzy metric spaces. Fixed Point Theory and Applications 2012 2012:225.

\section{Submit your manuscript to a SpringerOpen ${ }^{\circ}$ journal and benefit from:}

- Convenient online submission

Rigorous peer review

- Immediate publication on acceptance

- Open access: articles freely available online

- High visibility within the field

- Retaining the copyright to your article 